\title{
Análisis de la evolución de la idea de sostenibilidad en futuros maestros
}

\author{
María Carmen Conde Núñez \\ Avenida de la Universidad s/n, Departamento de Didáctica de las Ciencias \\ Experimentales y Matemáticas, Universidad de Extremadura, Cáceres. España. \\ cconde@unex.es \\ ORCID: https://orcid.org/0000-0003-1473-3531 \\ José Samuel Sánchez Cepeda \\ Avenida de la Universidad $s / n$, Departamento de Didáctica de las Ciencias \\ Experimentales y Matemáticas, Universidad de Extremadura, Cáceres. España. \\ samuel@unex.es \\ ORCID: https://orcid.org/0000-0001-8346-7236

\section{Aurora Muñoz-Losa} \\ Avenida de la Universidad $s / n$, Departamento de Didáctica de las Ciencias \\ Experimentales y Matemáticas, Universidad de Extremadura, Cáceres. España. \\ auroraml@unex.es \\ ORCID: https://orcid.org/0000-0003-1475-7031
}

[Recibido: 19 Julio 2019. Revisado: 20 Noviembre 2019. Aceptado: 13 Diciembre 2019]

Resumen: Hemos llevado a cabo una intervención con futuros maestros de Educación Primaria con la intención de trabajar el concepto de sostenibilidad. La secuencia desarrollada incluye la elaboración de un proyecto y una unidad didáctica por investigación escolar. A través de un portafolio los alumnos expresaron sus reflexiones sobre su idea de sostenibilidad. Para analizarlas se diseñó una tabla de niveles con indicadores. Más de la mitad del alumnado ha tenido una evolución significativa progresando desde ideas ligadas a aspectos físico-naturales exclusivamente, hacia un modelo emergente que incluye aspectos más sociales, complejos e interconectados.

Palabras clave Sostenibilidad; Medio ambiente; Formación de maestros; Evolución conceptual.

Article title: Analysis of the evolution in the idea of sustainability of future teachers after an intervention in the classroom

Abstract: We have carried out an intervention with future teachers of Primary Education with the intention of working on the concept of sustainability. The sequence includes the elaboration of a project and a didactic unit by school research. Through a portfolio the students expressed their thoughts on their idea of sustainability. To analyze them, a table of levels with indicators was designed. More than half of the students have had a significant evolution progressing from ideas linked to natural physical aspects exclusively, towards an emerging model that includes more social, complex and interconnected aspects

Keywords: Sustainability; Environment; Teacher Training; Conceptual evolution.

Para citar este artículo: Conde, M.C., Sánchez, J.S., y Muñoz-Losa, A. (2019) Análisis de la evolución de la idea de sostenibilidad en futuros maestros. Revista de Educación Ambiental y Sostenibilidad 1(2), 2203. doi:10.25267/Rev_educ_ambient_sostenibilidad.2019.v1.i2.2203

Revista de Educación Ambiental y Sostenibilidad

Universidad de Cádiz.ISSN: 2659-708X

http://dx.doi.org/10.25267/Rev_educ_ambient_sostenibilidad.2019.v1.i2.2203

https://revistas.uca.es/index.php/ReAys/index 


\section{Introducción}

Actualmente los desafíos ambientales son acuciantes y se hace necesario tomar medidas efectivas de inmediato. A la par, aparece también un futuro esperanzador donde los jóvenes de todo el mundo se manifiestan para poner freno al grave problema del cambio climático, exigiendo medidas políticas y de otros ámbitos que lo posibiliten.

En momentos así, donde los problemas están tan interconectados y donde la visión del medio ambiente y de la sostenibilidad ha ido evolucionando, entendemos que a nivel educativo tenemos un gran desafío.

Nuestro reto en este estudio es valorar cómo avanza la idea de sostenibilidad cuando trabajamos con alumnado del Grado en Educación Primaria, aunque somos conscientes de la limitación del tiempo en la etapa universitaria, que impide completar el proceso de maduración de la idea. La educación ambiental, de ahora en adelante EA, como proceso permanente a lo largo de toda la vida, seguirá haciendo que este cambio sea una realidad en todos los contextos. La sostenibilidad definirá una nueva forma de estar en el mundo desde una pertenencia más consciente y responsable, donde el cuidado hacia uno y de todas las formas de vida es esencial, porque forman parte de lo mismo. En esta evolución de la idea de sostenibilidad nos encontramos en la actualidad y todo ello debería tener cabida también en nuestras decisiones docentes.

Por todo ello nos planteamos con esta investigación conocer la idea de sostenibilidad que alcanzó el alumnado después de una intervención en la asignatura de 4o curso Conocimiento del Medio Natural en Educación Primaria. En esta asignatura, llevamos varios cursos trabajando una propuesta de investigación escolar con el alumnado, teniendo como referentes y materiales los trabajos de Rivero, Martín del Pozo, Solís y Porlán (2017). El tratamiento de la sostenibilidad se ha apoyado planteando la elaboración de un proyecto basado en un problema-pregunta ambiental relacionado directamente con el tema de su Unidad Didáctica, en adelante UD. Un portafolio elaborado por cada alumno al finalizar la asignatura nos sirvió para recoger los datos que nos muestran su idea de sostenibilidad después de la secuencia de enseñanzaaprendizaje.

\section{Marco teórico}

El término sostenibilidad suscita debates desde su origen. La conceptualización de esta idea ha ido evolucionando desde que apareció el término, según Gutiérrez, Benayas y Calvo (2006), en los debates institucionales de la Cumbre de Río (1992), y a partir del concepto "desarrollo sostenible", que aparece en el Informe Brundtland. De ahí hasta las últimas tendencias, que apuestan por sustituir ese término por una educación en y para el decrecimiento como alternativa (García Díaz, FernándezArroyo, Rodríguez Marín y Puig, 2019). Tal y como UNESCO (2012) propone, la sostenibilidad debe reformularse a medida que las realidades cambien y esto, independientemente del término que usemos, es lo que ha ido sucediendo.

Previamente, distintos autores han realizado estudios que recopilan la evolución y controversias alrededor de esta idea de sostenibilidad (Luffiego y Rabadán, 2000; Saura y Hernández, 2008; Bermejo, Arto, Hoyos y Garmendia, 2010; Gil y Vilches, 
2006; García Díaz et al. 2019) y también otros artículos que ayudan a ampliar la visión de qué es y hacia dónde ir (Aznar y Ull, 2009; Novo y Murga, 2010; Briceño y Equipo Área Educada, 2012; CADEP-CRUE, 2012).

Según Caride y Meira (2001), hablar de sostenibilidad es hablar de un concepto polisémico, para el que no existía un consenso sobre su significado. Gil y Vilches (2006, p. 515) propusieron que "sería preciso salir al paso de las discrepancias y reticencias que pueden bloquear una acción concertada: con matices, con lógicas variaciones semánticas y conceptuales en quienes proceden de distintos ámbitos".

Compartimos la idea con estos autores de que se trata de un concepto polisémico y en constante evolución para nosotros, en el que todos los cuestionamientos son útiles para avanzar en la evolución de la idea y, con ello, del compromiso ambiental. En este trabajo, seleccionamos algunas referencias sobre esta evolución de las últimas dos décadas, años en los que se ha constatado una reflexión más profunda y crítica del término, lo que nos ayudará también a conectar con la investigación que se presenta. El orden tratará de ser temporal en la medida de lo posible, entendiendo que esto no es algo lineal y que a veces aparece una idea que solo años después es madurada.

- De lo físico-natural a lo ambiental y hacia un modelo emergente. García Díaz (2002) recoge paradigmas y tendencias diferentes para enfocar la EA ligadas a la evolución del concepto de sostenibilidad. Así, plantea un modelo inicial naturalista, otro aún predominante unido a la protección y conservación del medio y, por último, un modelo emergente con indicadores de cambio social, enfocado en logros éticos y desarrollo personal, cuestionando o no el modelo de desarrollo establecido. Saura y Hernández (2008) plantean igualmente estas etapas.

- De la sostenibilidad débil a la fuerte. Luffiego y Rabadán (2000) consideraban que existían dos planteamientos claramente diferenciados: la sostenibilidad débil como una visión más antropocéntrica, compatible con el crecimiento y que aboga por la viabilidad del sistema socio económico con un planteamiento del medio ambiente localista; por otro lado, la sostenibilidad fuerte, con una visión más ecocéntrica, incompatible con el crecimiento y con un planteamiento del medio ambiente global y sistémico.

- Hacia los Valores éticos en la sostenibilidad. La Carta de la Tierra en su preámbulo aporta: "Necesitamos urgentemente una visión compartida sobre los valores básicos que brinden un fundamento ético para la comunidad mundial emergente". En su apartado final dice "Que el nuestro sea un tiempo que se recuerde por el despertar de una nueva reverencia ante la vida; por la firme resolución de alcanzar la sostenibilidad; por el aceleramiento en la lucha por la justicia y la paz y por la alegre celebración de la vida."

- Ética del cuidado. Ya en el 2000, la Carta de la Tierra introdujo una serie de valores éticos que se refieren al cuidado en su más amplio sentido. Para Comins (2018), las mujeres, en el marco de su histórica socialización como cuidadoras, han cultivado y desarrollado la lógica de la sostenibilidad de la vida. La sabiduría desarrollada por las mujeres para el sostenimiento de la vida es un legado que debería reconocerse y universalizarse, más allá de los roles de género, como valor humano. Novo y Murga (2010) una de las pautas educativas significativa que proponen es experimentar el valor del cuidado de 
otros (personas y naturaleza) como una cualidad específica de lo local que tiene repercusiones planetarias.

- Decrecimiento. Latouche (2006) habla de que la opción de cambiar el bientener por el bien-estar es difícil, porque el sistema actual no lo va a permitir, y no puede hacerse por imperativo. Para él, hay que extender la idea de que menos no es sinónimo de peor. Las posturas personales de apostar por un cambio y ejercerlo no serán suficiente, pero es una fuerza nada desdeñable. Bermejo et al. (2010) recopilan la evolución de la concepción de decrecimiento sostenible. Significa alcanzar un estado estacionario después de haber hecho una reducción equitativa del consumo.

- Ciudadanía planetaria. Lo esencial para Novo (2006) es el sentido de pertenencia, un sentimiento que configura una manera de ver el mundo y de situarse en él como sujeto de derechos y deberes. Posteriormente, Novo y Murga (2010, p. 184) recogen de otros autores varios matices que completan la idea de ciudadanía planetaria y que para ellas "acentúa la comprensión del ser humano como un ser ecodependiente que reconoce el valor intrínseco de lo vivo."

- [R]evolución cultural, educativa, tecnocientífica y política. Vilches, Gil, Toscano y Macías (2014), al finalizar la Década de la Educación para el Desarrollo Sostenible, hablan de una grave situación para la que aún se está a tiempo de actuar con un conjunto de acciones tecnocientíficas, legislativas y educativas, orientadas a hacer posible la superación del actual sistema socioeconómico, dando paso a la transición a la Sostenibilidad.

- Sostenibilidad de la trama de la Vida y Educación Ecosocial. Gutiérrez Bastida (2019) dice que hay muy poca cultura de la sostenibilidad. Propone una nueva ética ecosocial que respete, cuide y garantice la sostenibilidad de la trama de la Vida. Herrero (2018) en el mismo sentido habla de alternativas socioecológicas en la transición a la sustentabilidad, con propuestas ecofeministas para transitar a un mundo más justo y sostenible.

- La inevitabilidad del decrecimiento. Para Rodríguez-Marín, Fernández y García Díaz (2015), los "decrecentistas" no proponen ir hacia el decrecimiento sino dar una respuesta al mismo. Para García Díaz et al. (2019) el problema es que los partidarios de la Educación para el Desarrollo Sostenible, aunque insisten en la necesidad y urgencia de transformar las sociedades actuales hacia otros modelos sociales, no terminan por definir su posición ante el capitalismo. Son críticos con el término sostenibilidad que consideran incompatible con decrecimiento y ecocentrismo. Proponen incluso abandonar el término o usarlo en referencia a la supervivencia de la especie.

Desde Novo (1986) a la actualidad, la idea de sostenibilidad plantea aún debate y controversias. En cualquiera de los casos, se asume que estamos en un periodo de Transición ecológica (Novo, 2018), donde no solo se han de tomar medidas técnicas y económicas, que son imprescindibles, sino también proponer cambios profundos en el imaginario colectivo teniendo en cuenta lo anterior. Apuesta por la imaginación y la creatividad, por estimular la lucidez e ilusión, ensayando formas de vida que nos permitan ser más felices con menos cosas materiales y haciéndolo de forma cooperativa, donde la EA será un potente instrumento para esta transición. 
Todo lo visto hasta aquí nos parece un gran reto para ser reflexionado, adaptado e integrado en todo el sistema educativo. Este debate y las propuestas para integrarlo vienen siendo trabajadas desde hace tiempo en la Universidad (Gutiérrez-Pérez y Perales-Palacios, 2012; Geli, Collazo y Pons, 2019; García-González, Jiménez-Fontana y Cardeñoso (2017); García-González, Jiménez-Fontana y Azcárate, 2018). La CRUE nos aporta una definición de sostenibilidad que, recogiendo parte de la definición de UNESCO, se amplia de forma consensuada, abriendo caminos en esta construcción de la idea. La recogemos en último lugar por ser una propuesta que hasta ahora ha servido para aportar mucha luz en la integración de la sostenibilidad en la Universidad en España, el ámbito en el que se desarrolla nuestro trabajo: "La sostenibilidad es un concepto que incluye la búsqueda de la calidad ambiental, la justicia social y una economía equitativa y viable a largo plazo. Define un conjunto de criterios orientados al comportamiento ético con todo lo que nos rodea (recursos, personas, espacios...), de modo que permita lograr una equidad intra e intergeneracional, así como gestionar las relaciones con el medio natural y social, manteniendo su disponibilidad y equilibrio ecológico, y promoviendo una distribución más equitativa y justa de los recursos, beneficios y costes ambientales." (CADEP-CRUE, 2012, p.5).

Hoy, hablar de sostenibilidad es hablar de una idea mucho más compleja que abarca cuestiones clave de toda índole, que harán posible ese cambio de paradigma o nueva forma de estar en el mundo, quizá la única posible para la supervivencia de la especie.

\section{Objetivos}

- Diseñar una tabla de indicadores por niveles en los que incluir los aspectos que definan la idea de sostenibilidad que tienen los alumnos del último curso del Grado de Educación Primaria.

- Analizar el nivel alcanzado respecto a la idea de sostenibilidad por los alumnos después de una intervención en el aula de Conocimiento del Medio Natural en Educación Primaria.

\section{Desarrollo de la intervención}

Dedicamos todas las horas de seminario de la asignatura $(25 \%$ de un total de 6 créditos) al tema de sostenibilidad y al proyecto. Además, en las clases de gran grupo se dedicaron actividades en relación con la inclusión de la idea de sostenibilidad en su UD.

El proceso seguido para trabajar la sostenibilidad tuvo tres etapas principales tratando de combinar distintas estrategias que hicieran posible atender a los diversos contenidos según procedimientos coherentes entre sí, idea también reforzada por Pozuelo y Rodríguez (2008) al hablar del trabajo por proyectos. En su línea, presentamos una propuesta plural y compatible, dentro de los mismos principios y fundamentos:

1. En consonancia con el enfoque constructivista de la asignatura, comenzamos con una detección de ideas previas a través de una lluvia de ideas sobre la crisis ambiental. Posteriormente se realizó un seminario introductorio sobre medio ambiente, sostenibilidad y EA. Una vez presentado, los alumnos respondieron a un cuestionario para valorar la incorporación de la 
Sostenibilidad en su UD inicial. Pretendíamos que fueran conscientes de su situación de partida respecto del tema.

2. Acudimos a la metodología de aprendizaje basado en proyectos, con un trabajo colaborativo a través de un proyecto que implica planificación, argumentación y responsabilidad de todos. El objetivo era que se implicaran de forma activa en la indagación sobre un problema ambiental que les resultara cercano y motivador. Logramos que se plantearan hipótesis que respondieran a esa pregunta inicial y que estas guiaran la búsqueda de su información, la organización y la interpretación de los datos, hasta llegar a conclusiones que se expondrían en clase. A la par íbamos aportando material digital, lectura complementaria de documentos como la Carta de la Tierra y otras referencias sobre la problemática ambiental actual (Novo y Murga, 2010; Vilches y Gil, 2010) además de material didáctico relacionado. Se trabajaron los hábitos y actitudes personales. Con la intención de incardinar el proyecto y la UD de modo que la sostenibilidad quedara integrada en la misma, una de las preguntas de la trama conceptual debería estar ligada al problema ambiental objeto de estudio.

3. Finalizando el curso elaboraron tres actividades de reflexión sobre lo trabajado en torno a la sostenibilidad. Dos de carácter individual (portafolio y cuestionario en la plataforma Google Forms) y otra grupal (guía de reflexión).

El portafolio, fuente principal de datos de este estudio, se dividía en 5 bloques, siendo uno de ellos el de sostenibilidad. En cada bloque del portafolio debían resaltar las reflexiones, asociaciones, dudas, dificultades, situaciones, pensamientos, sentimientos, etc., que todo lo trabajado durante la asignatura hubiera generado en el alumno, incidiendo en que destacaran el aprendizaje alcanzado en relación con el tema, enlazándolo con su experiencia personal.

\section{Metodología}

La metodología seguida tiene carácter cualitativo de corte descriptivo e interpretativo. Hemos llevado a cabo un estudio de casos en dos grupos de clase que trabajan con el proceso anteriormente explicado. La docencia se lleva a cabo por dos profesoras diferentes que han trabajado de forma coordinada a lo largo del proceso.

Tomamos los datos revisando el portafolio elaborado por los alumnos de forma individual Para llevar a cabo el análisis de los datos a través de un análisis de contenido, se analizaron las aportaciones sobre su idea de sostenibilidad, un primer análisis sirvió para generar niveles que definían ideas clave en función de sus respuestas, diseñando una tabla en la que se diferenciaban aspectos clave del propio origen y evolución del término. A partir de ella, se realizó el vaciado de la información de sus portafolios, a la par que se continuó con la revisión bibliográfica y la validación por parte de expertos de distintos tipos. Con ello se fueron reformulando los distintos niveles hasta su diseño definitivo. Se realizaron revisiones en distintos momentos y por más de un investigador hasta lograr consenso, tanto para establecer los niveles, como para situar en ellos las reflexiones concretas del alumnado. La validación se llevó a cabo por distintos expertos en EA y sostenibilidad, unos ligados a la Universidad (en concreto a la Didáctica de las Ciencias Experimentales), y a otros contextos educativos y sociales. Los niveles definitivos se muestran en la Tabla 1. 
La muestra se compone de dos grupos del último curso del Grado de Educación Primaria en el curso 2017-18. Uno de los grupos, al que denominaremos LE por corresponder al itinerario de Lenguas Extranjeras, con 35 alumnos de la modalidad de Evaluación continua, y el otro con 75 alumnos, al que denominaremos EF por corresponder al itinerario de Educación Física, de los cuales 67 completaron el apartado de sostenibilidad. En ambos grupos había alumnos del itinerario generalista. Así, el total de alumnos que componen la muestra es de 102. Para organizar y analizar las aportaciones hemos codificado a los sujetos con números consecutivos y con el código identificativo del grupo al que pertenecen, así LE1-LE35 y EF1-EF67.

La tabla elaborada (ver Tabla 1) contiene 6 niveles de evolución de la idea de sostenibilidad. En cada nivel se parte de que el alumnado tiene integrado el nivel anterior y añade algún elemento significativo más en su reflexión que amplía su idea de sostenibilidad. Así, se parte de un nivel 0 en el que consideramos que no ha habido una evolución de su idea, al considerar solamente los aspectos físico-naturales del medio ambiente. En el nivel 1 relacionan los aspectos ambientales entendiendo como aspectos ambientales los económicos, sociales, y ecológicos entre otros. A partir de este nivel básico se añaden cuestiones como el compromiso personal y profesional en su labor como futuros maestros, conexiones entre elementos del medio ambiente, dimensión espacio-temporal, compromiso ético, decrecimiento, etc., hasta alcanzar el nivel 5.

Tabla 1. Tabla de niveles de evolución de la idea de sostenibilidad.

\section{NIVEL 0}

Los alumnos relacionan la sostenibilidad sólo con el medio natural.

\section{NIVEL 1}

Los alumnos relacionan los aspectos ambientales entendiendo como aspectos ambientales los económicos, sociales, y ecológicos entre otros.

\section{NIVEL 2}

Los alumnos relacionan los aspectos ambientales entendiendo como aspectos ambientales los económicos, sociales, y ecológicos entre otros.

Integran en su idea de sostenibilidad su responsabilidad personal, tanto sobre sus hábitos personales como en su relación con el medio.

Integran en su idea de sostenibilidad su responsabilidad en su labor como futuros maestros.

Expresión en su idea de sostenibilidad de conexiones existentes en el medio ambiente de forma básica (cada acción humana tiene una repercusión en el medio y sobre las personas).

\section{NIVEL 3}

Los alumnos relacionan los aspectos ambientales entendiendo como aspectos ambientales los económicos, sociales, y ecológicos entre otros.

Integran en su idea de sostenibilidad su responsabilidad personal, tanto sobre sus hábitos personales como en su relación con el medio.

Integran en su idea de sostenibilidad su responsabilidad en su labor como futuros maestros.

Tienen dimensión temporal (pasado, presente y futuro) y/o espacial (relación global-local)

Expresión en su idea de sostenibilidad de conexiones existentes en el medio ambiente con mayor nivel de complejidad (ecodependencia, interdependencia, ...). 


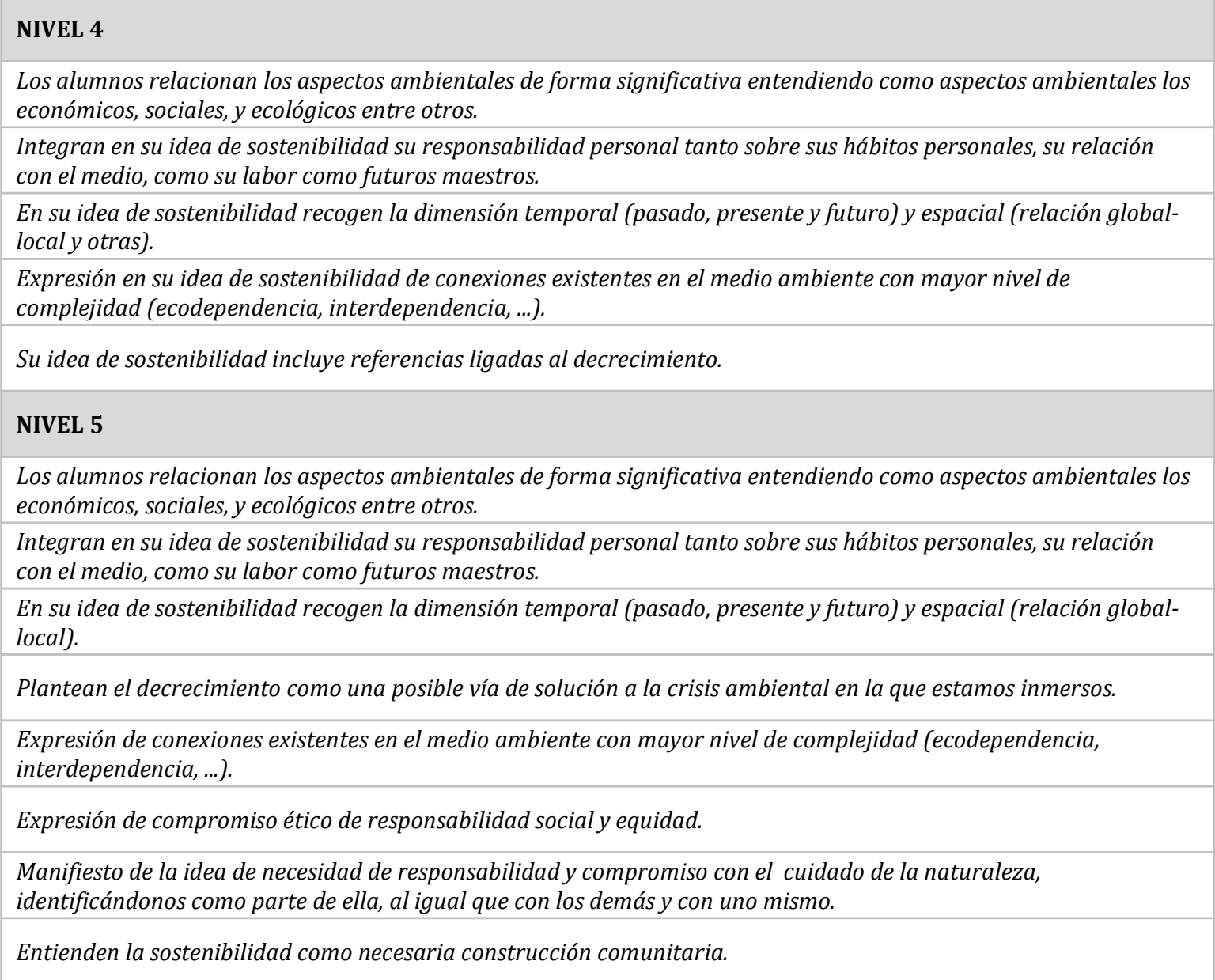

\section{Resultados, análisis y discusión}

Presentamos, por razones de espacio, algunas de las respuestas significativas del alumnado que nos ayuden a entender los distintos niveles de evolución o complejidad sobre la idea de sostenibilidad.

\section{Ideas previas sobre sostenibilidad}

A través de una lluvia de ideas con tan solo el grupo LE al inicio de curso, planteamos la siguiente cuestión ¿en qué pensáis cuando hablamos de crisis ambiental? Observamos como en todos los casos, lo que la definía tenía que ver exclusivamente con cuestiones sobre el medio físico natural: el cambio climático, pérdida de hábitats, vertidos contaminantes, residuos radiactivos, emisión de CFC, extinción de especies, aumento de gases contaminantes, aumento del consumo de carne, introducción de especies no autóctonas, incendios, erosión, deforestación, sequía, huracanes, terremotos, mala gestión de la caza, sobreexplotación.

Cuando se les plantea posteriormente ¿en qué pensáis cuando se habla de sostenibilidad? De forma grupal todas las respuestas van en la misma línea. Ej. "proteger la naturaleza para que las generaciones futuras puedan disfrutar de sus privilegios igual que nosotros". Observamos que, en su situación de partida, lo que define a lo ambiental y a la idea de sostenibilidad es el medio físico natural y la relación con él. Estos resultados concuerdan con los de la investigación de Briceño et al. (2012) ante alumnado y profesorado cuando se les pregunta qué entienden por 
sostenibilidad. Igualmente ocurre ante alumnado de Máster, según el estudio de García-González, Jiménez-Fontana y Cardeñoso (2017), donde la componente natural en su idea de sostenibilidad tiene una fuerte presencia antes y después de la intervención.

\section{Ideas sobre sostenibilidad al finalizar la intervención}

Su reflexión en el portafolio no está orientada según un cuestionario de preguntas, sino que es libre y recoge la maduración de una idea después de trabajar en ella un tiempo de 3 meses y medio. Presentamos los resultados agrupados por niveles con algunos ejemplos representativos.

\section{Alumnos en nivel 0}

Destacamos las frases de dos alumnos:

"En todos los años de educación nos han hablado e intentado concienciar sobre el cuidado y respeto del medio ambiente, desde acciones pequeñas en las aulas, como utilizar papel reciclado, reutilizar material, recoger la basura del patio, etc. hasta charlas y participación en proyectos de sostenibilidad, como por ejemplo hacer quedadas para recogida de basura por el pueblo, charlas sobre reciclaje y reutilización, concursos de murales sobre el respeto del medio ambiente, etc." (EF9)

"En la actualidad estamos viendo como nuestro planeta está cambiando debido al trato que nosotros los hombres le estamos dando para satisfacer nuestras necesidades. Un trato que ha sido arrasador y obviamente nada sostenible. Esto ha provocado la disminución en la biodiversidad, desaparición de grandes selvas, bosque, animales, etc. Todo esto es una muestra de que la humanidad no ha logrado construir con éxito un modelo que sea responsable para la conservación de los recursos naturales tanto renovables como no renovables que nos ofrece el planeta, sino que estamos arrasando el planeta poco a poco." (EF26)

Para gran parte del grupo, 34,3 \% (12 alumnos) en LE y 50,7 \% en EF (34 alumnos), no se ha asumido que la cuestión ambiental se refiere a mucho más que el medio físico natural. Si analizamos la definición de medio ambiente, para la RAE (Real Academia Española), es el "Conjunto de componentes físicos, químicos y biológicos, externos con los que interactúan los seres vivos". De hecho, el término ambiental, aparece en documentos oficiales, y en referencias de muchos autores especializados en la cuestión para hacer alusión a la dimensión natural del término (tomando el todo como una de las partes).

Nosotros en la intervención de clase nos acogíamos a la idea de medio ambiente como "sistema constituido por factores físicos y socio-culturales interrelacionados entre sí, que condicionan la vida de los seres humanos a la vez que son modificados y condicionados por éstos", definición de Novo (1986) coincidente con la de (UNESCO, 2012). Esta última nos parece una idea mucho más integradora que la que ofrece la RAE, aceptada en el campo de la EA desde hace mucho tiempo, y que nos puede ayudar a entender mejor las conexiones. Sin embargo, esta conceptualización es difícil de incorporar a sus esquemas de conocimiento.

Evidentemente el término ambiental sigue arraigado a lo físico-natural y a la hora de avanzar hacia una idea de sostenibilidad, como era el caso, aún después de haber aclarado a qué idea de medio ambiente nos acogíamos sigue existiendo una tendencia 
fuerte que hace que aparezca esta concepción alternativa, que les lleva de nuevo a relacionar el medio ambiente sólo con el medio físico y natural. Esto puede dificultar la evolución hacia una conceptualización más amplia del término sostenibilidad, cuando ni siquiera se ha avanzado en la conceptualización sobre medio ambiente y lo ambiental. Ello puede tener un origen cultural por su calado en la sociedad, su permanencia en el mundo educativo ambiental, generándose ambigüedad que podría dificultar la progresión a la hora de ir ampliando la visión sobre la idea de sostenibilidad.

Este nivel 0 coincidiría con lo que para García Díaz (2002) y Saura y Hernández (2008) son las dos primeras etapas en la evolución del concepto ligadas a aspectos físico naturales o de conservación y protección de los recursos naturales, de la fauna y de la flora.

Creemos que la diferencia entre ambos grupos puede deberse a que en LE se hizo la lluvia de ideas y la puesta en común para saber qué entendían por crisis ambiental y por sostenibilidad en el inicio. Hacer explícitas sus ideas previas en este sentido, y reflexionar sobre su lugar de partida, les sirvió para que evolucionaran en su mayoría a un nivel más alto, a diferencia del grupo EF, donde no se llevó a cabo. Por este motivo, esta idea alternativa tan potente permaneció en el 51 \% del grupo EF.

\section{Alumnos en nivel 1}

Destacamos la contribución de dos alumnos en el nivel 1.

"A medida que íbamos avanzando en la realización del proyecto, nos dimos cuenta de que debíamos analizar no solo el aspecto económico de las posibles soluciones que propusimos, sino que también teníamos que tener en cuenta las consecuencias ambientales, políticas, sociales y culturales que suponía la realización de nuestro proyecto. Por todo esto, comprendimos que el significado de sostenibilidad no se refiere únicamente al ámbito económico, sino que engloba todos los aspectos mencionados anteriormente." (EF59)

"El desarrollo sostenible debe plantar cara a grandes problemas a nivel global que deben solucionarse si realmente queremos conseguir una sociedad más sostenible: por ejemplo, el efecto invernadero o la destrucción de la capa de ozono en términos ambientales, el acceso al agua potable o la superpoblación en términos sociales..." (LE30)

Un 34,3 \% de alumnos de LE (12) y un 35,8 \% de EF (24) han alcanzado el nivel 1. Estos alumnos han sido capaces de avanzar consolidando una visión de lo ambiental en la que confluyen los aspectos naturales, económicos, socio culturales... Aunque no recogieran una definición del medio ambiente amplia, sí acogen varios de estos aspectos en su idea de sostenibilidad, por lo que consideramos que su idea ha evolucionado. Es una fase intermedia en la que la sostenibilidad va más allá para el alumnado del medio natural, es decir del modelo inicial o de corte naturalista mencionado por García Díaz (2002).

\section{Alumnos en nivel 2}

Extraemos reflexiones de dos alumnos en el nivel 2.

"Lo que se pretende es que los niños y niñas desde su educación más temprana comprendan que la sostenibilidad se encuentra en todos los ámbitos de su vida 
cotidiana y que no se trata (solo) de cuidar el medio ambiente. Gracias a este tema me he hecho preguntas $y$ he investigado. Así hemos descubierto algunos programas llevados a cabo para fomentar la sostenibilidad (...) Con nuestras acciones decidiremos cuál será la escena de futuro." (LE6)

“(....)Enormes desajustes entre países, gran aumento de la contaminación, emigraciones en masa por la huida de guerras y de su situación en los países donde existe una gran pobreza, desestabilizando los sistemas de los países desarrollados y las grandes ciudades y pueblos. Además del calentamiento global y sus consecuencias, falta de sanidad y educación etc. (...) Por eso mismo es nuestra responsabilidad, la de todos, intentar colaborar para que eso no ocurra. Para ello debemos educar a las nuevas generaciones desde la base, y hacerles conscientes de que el mundo está realmente en peligro e intentar que ellos puedan mejorar el planeta y conseguir un desarrollo sostenible." (EF66)

Un 14,3\% (5) de LE y un 11,9 \% (8) de EF han alcanzado el siguiente paso en la evolución de esta conceptualización: la integración de la responsabilidad personal propia, que en caso de nuestra experiencia ha ido también ligada a que los alumnos expresan su papel como futuros maestros en esta cuestión. Quizá la orientación práctica con la que lo abordamos en clase les ha hecho conscientes de ello y de sus posibilidades sea cual sea el tema que se aborde. Sentirse co-responsables de la situación ambiental y con oportunidades para cambiarla es ya no vivir el medio como algo externo. También en este nivel se han valorado las expresiones sobre las conexiones existentes entre los distintos elementos que constituyen lo ambiental. Este nivel es más coincidente con lo que García Díaz (2002) definía como modelo ambientalista en educación ambiental.

\section{Alumnos en nivel 3}

Igualmente presentamos las aportaciones de dos alumnos.

"Sé que era injusto y cómodo mi pensamiento, pensar que los grandes problemas del mundo, aunque nos estén afectando, son culpas de otros (...) Gracias al proyecto de sostenibilidad, no solo he cambiado mi idea sobre el tema que he trabajado, sino que he abierto los ojos ante el mundo y sus problemas. Voy interesándome no solo por lo lejano, sino también por lo cercano. He empezado con la mina de litio investigando sobre la verdad, sobre cómo afectaría y sobre cómo hacer para que los niños del futuro la conozcan." (LE27)

"Me fui dando cuenta y razonando que la sostenibilidad era mucho más que un conjunto de conocimientos inconexos que tampoco tenían demasiada cercanía con nuestra problemática actual, así tras esto, puedo afirmar que para mí la sostenibilidad está presente en todos los ámbitos, es decir no solo afecta a la economía mundial, sino también a la política, a la educación e incluso a la salud." (LE29)

Un 17,1 \% (6) de LE y ninguno de EF alcanzaron el nivel 3. De alguna forma el alumnado que llega a este nivel empieza a hablar de las dimensiones temporal (pasado, presente y futuro) y/o espacial (relación global-local), aunque no todos mencionan ambas a la vez. También expresan conexiones existentes en el medio ambiente. Algunos alumnos han alcanzado este nivel con un pensamiento más complejo, incluyendo la responsabilidad personal y social, con lo que sería un nivel de aproximación mayor que en el nivel 2, que estaba más ligado a las buenas prácticas. 
Las conferencias de expertos y ecologistas sobre el cuestionado proyecto de creación de una mina de extracción de litio en la ciudad de Cáceres, al que dedicamos tiempo conjunto ambos grupos, ayudaron a planteamientos locales. Una de las razones por las que más estudiantes no llegaron a este nivel, puede ser que el documental "Before the flood" (Di Caprio, 2018), tan solo se visualizó en el grupo LE, siendo un documental que ayudaba a alcanzar una visión global e interconectada. Creemos que a partir de este nivel las reflexiones se podrían situar en lo que García Díaz (2002) definía como modelo emergente en educación ambiental, más ligado ya al cambio social, además de al desarrollo sostenible.

\section{Alumnos en nivel 4}

Tan solo un alumno perteneciente al grupo de EF alcanzó, a nuestro juicio, el nivel 4.

"Resulta llamativo la utilización de la palabra necesidad. Entendemos por necesario algo que es insustituible en nuestra vida, como es el agua, el oxígeno... pero no lo es, por ejemplo, disfrutar de alimentos que se encuentran fuera de temporada. Aquí es donde radica el problema por el que surge el concepto de sostenibilidad y en consecuencia del desarrollo sostenible. El ser humano no es capaz de distinguir entre querer poseer un elemento o realmente necesitarlo. Esto deriva en una de las peores consecuencias para el planeta Tierra, la creación de un consumo negativo.

(...) El objetivo principal que debemos establecer como docentes es concienciar a los alumnados sobre los problemas ambientales y del desarrollo en su globalidad, despertando el interés por actuar. En relación a la necesidad de cambio, son muchos los objetivos que podemos plantear a nuestros alumnos. Estos se pueden introducir mediante acciones como reducir, reutilizar y reciclar recursos, contribuir a la educación y acción ciudadana...

Es fundamental que los alumnos comprendan que existe una cadena en cuyos extremos de sus eslabones encontramos los problemas a nivel mundial y local. Es así como relacionarán el conjunto de problemas locales o próximos como un producto que nos lleva a los problemas de nivel mundial." (EF44)

Captar el concepto de verdaderas necesidades es algo clave y este alumno lo ha logrado expresar, coincidiendo con la evolución histórica del concepto de desarrollo sostenible. Las primeras ideas de decrecimiento son expresadas por él como "consumo negativo", queriendo referirse a la idea de agotamiento. La referencia al decrecimiento es aún incipiente, pero ayudan a llegar a él. En nuestra intervención no hubo posibilidad de tratar esta cuestión con mayor profundidad por falta de tiempo. Nos parece que requiere una secuencia reflexiva, dialógica y no aleccionadora, en este sentido.

\section{Alumnos en nivel 5}

Por último, ningún alumno ha llegado al nivel máximo de evolución de referencia donde se incluye la expresión de un compromiso de responsabilidad social marcadamente ético y de equidad. Otra de las cuestiones que define el nivel 5 es el manifestar la necesidad de respeto y cuidado tanto hacia uno mismo como hacia los demás compatible con el respeto y cuidado a la naturaleza identificándose como parte de ella. En este sentido Novo y Murga (2010) aportan una visión de la relación ser humano/naturaleza que tiene enfoque sistémico. 
La deseable profundización sobre estas cuestiones que no se han llegado a alcanzar en este nivel exigiría más tiempo y una secuencia constructivista más completa, de modo que se pudiera avanzar sobre aspectos que requieren mayor reflexión y una enseñanza-aprendizaje más dialógica.

\section{Conclusiones}

Respecto al primer objetivo, se ha logrado elaborar una tabla que nos ha servido para entender la progresión de los alumnos. En el proceso de validación se plantearon distintas opciones como la elaboración de niveles de progresión dentro de los indicadores de cada nivel (responsabilidad personal, conexiones existentes en el medio, etc.) lo que permitiría saber para cada indicador el grado de evolución en el que se encuentra cada persona. Hemos optado, sin embargo, por la opción que nos ofrecía extraer la maduración de la idea de sostenibilidad de tal manera que cada nivel incluía lo logrado en el nivel anterior, algún aspecto nuevo o un desarrollo más complejo de alguna de las cuestiones. Así, creemos que la tabla con niveles de progresión ha permitido recoger adecuadamente, y de forma más global el grado de evolución de sus ideas. No obstante, esta propuesta siempre debe ser revisable, tanto a nivel conceptual como en su diseño, siendo adaptable según los casos. Creemos que, estando en construcción, podría aprovecharse como propuesta para seguir discutiendo juntos investigadores, educadores y alumnado. De hecho, a la hora de validar la misma, han aparecido cuestiones interesantes que nos sugieren, en la diversidad de pensamientos al respecto, la oportunidad de ser un instrumento útil para la reflexión conjunta. Una propuesta abierta en permanente construcción para avanzar de forma dialógica sobre la idea de sostenibilidad.

Respecto al segundo objetivo, encontramos bastante coincidencia entre los niveles alcanzados por los alumnos que los que se han dado en la sociedad respecto a la idea de sostenibilidad, en los que los primeros niveles correspondían a visiones reduccionistas ligadas al medio físico-natural y a su conservación. Para nosotros, el $45 \%$ del alumnado que está en este nivel 0 , no ha experimentado una evolución significativa. Creemos que hay una idea alternativa potente tanto en el alumnado como en la sociedad, e incluso en instancias oficiales e investigadoras para los que el término ambiental sigue muy vinculado a las cuestiones del medio físico-natural. Esto dificulta en gran medida la progresión esperada. Es necesario tener en cuenta este aspecto cuando se trabaje para diseñar propuestas que faciliten la evolución esperada. Se observa cómo un $35 \%$ de alumnos llegaron al nivel 1, el 13\% al nivel 2 y el $6 \%$ al nivel 3. Solo un 1\%, esto es, un alumno alcanzó el nivel 4 y ninguno al nivel de referencia deseable. Que los alumnos lleguen a distintos niveles de complejidad coincide con los resultados de García-González, et al. (2017). También se aprecia la dificultad de lograr que la idea de sostenibilidad del alumnado evolucione desde una visión físico-natural al modelo emergente, con indicadores de cambio social, ahondando en aspectos más complejos y siempre interconectados, éticos, de cuidado, de equidad, de decrecimiento, etc. Por ello entendemos la necesidad de diseñar propuestas didácticas acordes a esta evolución.

Pensamos que se requiere más tiempo para interiorizar y madurar todas estas cuestiones y llevarlas a la vida más allá de esta etapa educativa. Nuestros planteamientos iniciales no contaban con que pudiéramos abordar las cuestiones del último nivel debido al tiempo disponible. 
Creemos necesario que el profesorado sea consciente de dónde parte respecto a esas mismas ideas antes de llevar a cabo la secuencia didáctica, cuál es su posicionamiento, así como el horizonte deseable. Seguramente, para todas las personas que intervienen en el proceso, incluido el profesorado, haya una evolución conjunta más significativa.

Al tratarse la sostenibilidad de una idea en evolución permanente y cuestionada incluso, es una oportunidad para trabajar con ello de forma dialógica. Así, aprovechamos los diferentes puntos de vista, para analizar y reflexionar conjuntamente sobre ello. La senda que hace unos años planteamos iniciamos (Conde, Moreira, Sánchez y Mellado, 2010) en la que la capacidad de imaginar y crear alternativas a un mundo tan complejo fuese objetivo esencial de la educación para la sostenibilidad, nos parece debe ser aún más fortalecida. Podemos aprovechar esta situación para seguir avanzando en el aprendizaje dialéctico, donde todos aprendemos de todos, en el papel de las emociones, no solo del conocimiento, y en la importancia de la interiorización y no solo la actividad, tal y como entonces planteamos.

A pesar de las limitaciones, encontramos que ha habido una maduración significativa por parte del alumnado a través de la intervención planteada, y con el uso del portafolio como instrumento para plasmarlo. La interiorización ha llevado a que el alumnado no diese definiciones "tipo libro" sobre sostenibilidad, sino que la integrara también con su propia persona y emociones.

Alcanzar un gran nivel de evolución sobre sostenibilidad requiere un trabajo conjunto desde las primeras etapas educativas. En este sentido, compartimos que la falta de trabajo sobre la idea de sostenibilidad se acusa desde la escuela (Marcén, 2017) y que continua hasta la Universidad (Geli et al. 2019). Para García-González, et al. (2018), la situación de crisis puede ser la coyuntura para acometer los cambios demandados por los procesos de enseñanza-aprendizaje universitarios. De ahí vemos la importancia de tenerla en cuenta en todas las etapas para lograr una evolución y maduración apropiada ante los nuevos retos. Es imprescindible trabajar estas cuestiones con los futuros docentes donde reside una de las claves que promoverá el cambio.

En este sentido, estamos trabajando en una propuesta a través de una secuencia constructivista en la que el aprendizaje dialógico y la reflexión sean más protagonistas.

\section{Referencias}

Aznar, P. y Ull, M. A. (2009). La formación de competencias básicas para el desarrollo sostenible: el papel de la Universidad. Revista de Educación, nº extr. 2009, 219237.

Bermejo, R., Arto, I., Hoyos, D. y Garmendia, E. (2010). Menos es más: del desarrollo sostenible al decrecimiento sostenible, Cuadernos de Trabajo de Hegoa, 52. Vitoria-Gasteiz:

UPV/EHU. http://publicaciones.hegoa.ehu.eus/uploads/pdfs/152/Cuaderno_de_trabajo_ 52.pdf?1488539563

Briceño, J. J. y Equipo Área Educada (2012). Retos para la sostenibilidad en el Área metropolitana del Valle de Aburrá de Antioquia Colombia. Percepciones acerca 
de lo que entendemos por este término y ejemplos de actividades educativas. Revista Eureka sobre Enseñanza y Divulgación de las Ciencias, 9(2), 278-293. https://doi.org/10.25267/rev_eureka_ensen_divulg_cienc.2012.v9.i2.10

CADEP-CRUE (2012). Directrices para la introducción de la sostenibilidad en el curriculum. Actualización de la declaración institucional de 2005. Recuperado de: http://www.crue.org/SitePages/Documentos-Sostenibilidad.aspx

Caride, J. A. y Meira, P. A. (2001). Educación ambiental y desarrollo humano. Barcelona: Ariel.

Comins, I. (2018). Ética del Cuidado: hacia una autoconciencia ampliada. Cuadernos de Pedagogía, 485, 1-2.

Conde, M. C., Moreira, A, Sánchez, J. S. y Mellado, V. (2010). Una aportación para las "Escuelas Sostenibles" en la Década de la Educación para el Desarrollo Sostenible. Revista Eureka sobre Enseñanza y Divulgación de las Ciencias, 7(no extr.), 363-373.

Di Caprio, L. (2018). Video en: https://www.youtube.com/watch?v=8UqBuUSn3hY

García Díaz, J. E. (2002). Los problemas de la Educación Ambiental: ¿Es posible una Educación Ambiental Integradora? Investigación en la Escuela, 46, 5-25.

García Díaz, J. E., Fernández-Arroyo, J., Rodríguez-Marín, F. y Puig Gutiérrez, M. (2019). Más allá de la sostenibilidad: por una educación ambiental que incremente la resiliencia de la población ante el decrecimiento/colapso. Revista de Educación $\begin{array}{llll}\text { Ambiental } \quad y & \text { Sostenibilidad, } & 1101 .\end{array}$ https://doi.org/10.25267/Rev_educ_ambient_sostenibilidad.2019.v1.i1.1101

García-González, E., Jiménez-Fontana, R. y Cardeñoso, J. Ma (2017). La Educación para la Sostenibilidad. Una experiencia formativa posgrado para futuros profesionales de la educación. En VII Congreso Universidad y Cooperación al Desarrollo. Madrid:

UAM.

http://congresos.fuam.es/imagenes/descargas_archivos/Libro\%20Guia \%20FINAL_111.pdf

García-González, E., Jiménez-Fontana, R. y Azcárate, P. (2018). La formación de maestros en Educación Infantil desde la perspectiva de la sostenibilidad. Un estudio de caso en la Universidad de Cádiz. Revista Qurriculum, 31, 31-56. http://doi.org/10.25145/j.qurricul.2018.31.002

Geli, A. M., Collazo, L.M. y Pons, I. M. (2019). Contexto y evolución de la sostenibilidad en el curriculum de la universidad española. Revista de Educación Ambiental y Sostenibilidad, $1(1)$, 1102. https://doi.org/10.25267/Rev_educ_ambient_sostenibilidad.2019.v1.i1.1102

Gil, D. y Vilches, A. (2006). Algunos obstáculos e incomprensiones en torno a la Sostenibilidad. Revista Eureka sobre Enseñanza y Divulgación de las Ciencias, 3(3), 507-516. Recuperado a partir de https://revistas.uca.es/index.php/eureka/article/view/3854

Gutiérrez, J., Benayas, J. y Calvo, S. (2006). Educación para el Desarrollo Sostenible: Evaluación de retos y oportunidades del Decenio 2005-2014. Revista Iberoamericana de Educación, 40, 25-69. https://doi.org/10.35362/rie400781 
Gutiérrez-Pérez, J., y Perales-Palacios, F. J. (2012). Ambientalización curricular y sostenibilidad. Nuevos retos de profesionalización docente. Profesorado: Revista de currículum y formación del profesorado, 16(2), 1-10. https://recyt.fecyt.es/index.php/profesorado/article/view/43676

Gutiérrez Bastida, J. M. (2019). 50 años de Educación Ambiental: un balance incompleto hacia la educación ecosocial en el Antropoceno. Carpeta Informativa del CENEAM, Mayo 2019, 1-13. https://www.miteco.gob.es/es/ceneam/articulos-de-opinion/2019-05-gbastida_tcm30-496628.pdf

Herrero, Y. (2018). Video en: https://www.youtube.com/watch?v=Eq-jysIgnIs

Latouche, S. (2006). La apuesta por el Decrecimiento. Barcelona: Icaria.

Luffiego, M. y Rabadán, J. M. (2000). La evolución del concepto de Sostenibilidad y su introducción en la Enseñanza. Enseñanza de las Ciencias, 18(3), 473-486.

Marcén, C. (2017). Medioambiente y Escuela. Zaragoza: Octaedro.

Novo, M. (1986). La Educación Ambiental: bases éticas, conceptuales y metodológicas. Madrid: Universitas.

Novo, M. (2006). El desarrollo sostenible: su dimensión ambiental y educativa. Madrid: Pearson Educación.

Novo, M. (2018). Educación ambiental y transición ecológica. AMBIENTA, 125, 32-41. https://sites.google.com/gl.miteco.es/ambienta/n\%C3\%BAmerosanteriores/125/125-a4

Novo, M. y Murga, M. A. (2010). Educación ambiental y ciudadanía planetaria. Revista Eureka sobre Enseñanza y Divulgación de las Ciencias, no extr. 2010, 179-186. https://www.redalyc.org/pdf/920/92013009003.pdf

Pozuelos, F. J., Rodríguez, F. de P. (2008). Trabajando por proyecto en el aula. Aportaciones de una investigación Colaborativa. Investigación en la escuela, 66, 52-57.

Rivero, A., Martín del Pozo, R., Solís, E. y Porlán, R. (2017). Didáctica de las Ciencias Experimentales en Educación Primaria. Madrid: Síntesis.

Rodríguez-Marín, F., Fernández Arroyo, J. y García Díaz, J. E. (2015). El huerto escolar ecológico como herramienta para la educación en y para el decrecimiento. Investigación en la Escuela, 86, 35-48.

Saura, P. y Hernández, M. A. (2008). La evolución del concepto de Sostenibilidad y su incidencia en la Educación ambiental. Teoría de la Educación, Rev. Interuniversitaria, 20, 179-204.

UNESCO (2012). Forjar la educación del mañana. Decenio de las Naciones Unidas de la Educación para el Desarrollo Sostenible - Informe 2012 (abreviado). https://es.unesco.org/themes/educacion-desarrollo-sostenible/comprenderEDS/decenio-onu

Vilches, A. y Gil, D. (2010). ¿Cómo puede contribuir la educación a la construcción de un futuro sostenible? Revista Eureka sobre Enseñanza y Divulgación de las 


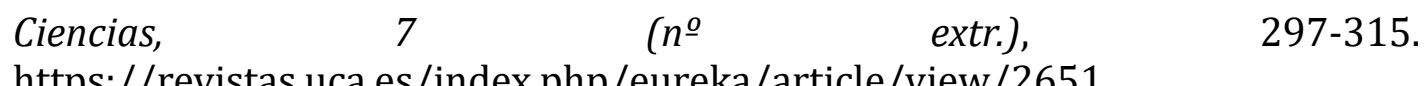
https://revistas.uca.es/index.php/eureka/article/view/2651

Vilches, A., Gil, D., Toscano J. C. y Macías O. (2014). La transición a la Sostenibilidad (o Sustentabilidad) como [r]evolución cultural, educativa, tecnocientífica y política. OEI: https://www.oei.es/historico/decada/accion1_contenido.php 